Electric-field induced quantum broadening of the characteristic energy level of traps in semiconductors and oxides

Mazharuddin Mohammed, Anne S. Verhulst, Devin Verreck, Maarten Van de Put, Eddy Simoen, Bart Sorée, Ben Kaczer, Robin Degraeve, Anda Mocuta, Nadine Collaert, Aaron Thean, and Guido Groeseneken

Citation: J. Appl. Phys. 120, 245704 (2016); doi: 10.1063/1.4972482

View online: http://dx.doi.org/10.1063/1.4972482

View Table of Contents: http://aip.scitation.org/toc/jap/120/24

Published by the American Institute of Physics 


\title{
Electric-field induced quantum broadening of the characteristic energy level of traps in semiconductors and oxides
}

\author{
Mazharuddin Mohammed, ${ }^{1,2, a)}$ Anne S. Verhulst, ${ }^{1}$ Devin Verreck, ${ }^{1,2}$ Maarten Van de Put, ${ }^{1,3}$ \\ Eddy Simoen, ${ }^{1}$ Bart Sorée, ${ }^{1,2,3}$ Ben Kaczer, ${ }^{1}$ Robin Degraeve, ${ }^{1}$ Anda Mocuta, ${ }^{1}$ \\ Nadine Collaert, ${ }^{1}$ Aaron Thean, ${ }^{1, b)}$ and Guido Groeseneken ${ }^{1,2}$ \\ ${ }^{1}$ imec, Kapeldreef 75, 3001 Leuven, Belgium \\ ${ }^{2}$ Department of Electrical Engineering, KU Leuven, 3001 Leuven, Belgium \\ ${ }^{3}$ Department of Physics, Universiteit Antwerpen, 2020 Antwerpen, Belgium
}

(Received 30 September 2016; accepted 6 December 2016; published online 23 December 2016)

\begin{abstract}
The trap-assisted tunneling (TAT) current in tunnel field-effect transistors (TFETs) is one of the crucial factors degrading the sub- $60 \mathrm{mV} / \mathrm{dec}$ sub-threshold swing. To correctly predict the TAT currents, an accurate description of the trap is required. Since electric fields in TFETs typically reach beyond $10^{6} \mathrm{~V} / \mathrm{cm}$, there is a need to quantify the impact of such high field on the traps. We use a quantum mechanical implementation based on the modified transfer matrix method to obtain the trap energy level. We present the qualitative impact of electric field on different trap configurations, locations, and host materials, including both semiconductors and oxides. We determine that there is an electric-field related trap level shift and level broadening. We find that these electricfield induced quantum effects can enhance the trap emission rates. Published by AIP Publishing.

[http://dx.doi.org/10.1063/1.4972482]
\end{abstract}

\section{INTRODUCTION}

The inherent band to band tunneling (BTBT) principle in tunnel field-effect transistors (TFETs) can alleviate the sub-threshold swing (SS) limit of MOSFETs for low-power applications. ${ }^{1,2}$ Due to the usual low on-currents in $\mathrm{Si}$ TFETs, an extensive study is ongoing in pursuit of alternative device materials and structures whereby the heterostructures look quite promising. ${ }^{3-8}$ However, new materials and heterostructures may result in significant trap concentrations at semiconductor-dielectric interfaces or heterostructure interfaces. In fact, the SS degradation in TFETs is presently associated with trap-assisted tunneling. ${ }^{9}{ }^{910}$ In MOSFETs, the impact of trap-assisted tunneling (TAT) is also noticeable in Stress-Induced Leakage Currents (SILC) ${ }^{11}$ and in GateInduced Drain Leakage (GIDL). ${ }^{12}$ Therefore, an in-depth understanding of TAT in semiconductor devices is essential to assess the device capabilities. This requires sufficiently accurate theoretical models.

While the external influence of electric field on the TAT has been studied rigorously, its intrinsic impact on the trap characteristics has received less attention. ${ }^{13,14}$ Since the electric field in TFETs can readily reach beyond $1 \mathrm{MV} / \mathrm{cm}$ in both semiconductor and oxide, we explore the implications of such high electric field on the trap energy level and its possible effects on the emission rates. In particular, we investigate the significance of the trap level shift and broadening.

The presence of traps establishes a band discontinuity on the sub-microscopic scale. ${ }^{15}$ The corresponding electrostatics is similar to that of heterostructures. Hence, the trap

\footnotetext{
${ }^{\text {a) }}$ Mazharuddin.Mohammed@imec.be

${ }^{b} A$. Thean was with imec during the research and is currently affiliated with National University of Singapore (NUS), Singapore.
}

system in semiconductor devices can conveniently be configured as a quantum well in the electrostatic potential. Analogous to the determination of quasi-bound and resonant states of semiconductor heterostructures, we will determine the characteristic trap energy level in the presence of an electric field by a quantum mechanical implementation based on the transfer matrix method. The content of this paper is organized as follows. In Section II, we present the formalism and the numerical implementation for trap level extraction. The impact of electric field on semiconductor traps and on different trap configurations is presented in Section III. Section IV considers the impact on the oxide trap characteristics. The electric field impact on the emission rates of semiconductor and oxide traps is discussed in Section V. Finally, we conclude this paper in Section VI.

\section{FORMALISM AND NUMERICAL PROCEDURE}

Over the years, numerous approaches have been proposed to find the quasi-bound and the resonant states of heterostructures. These approaches can be broadly classified as "large matrix," involving a full system Hamiltonian, ${ }^{16,17}$ and "small matrix," based on the transfer method. ${ }^{18}$ Among the small matrix based approaches, the modified transfer matrix (MTM) method is preferred for our investigation of the electric field effect on the trap level in a one dimensional structure. ${ }^{19}$ The choice for the MTM method is based on its computationally efficient numerical implementation. In addition to this, the abrupt transition of the electrostatic potential at a heterointerface, which will be used to configure the trap, can be handled efficiently. However, the extension of the MTM method to higher dimensional (2D or 3D) heterostructures, which are beyond the scope of this article, is uncer$\operatorname{tain}^{20}$ and hence other approaches may be required. In this article, the different cases of semiconductor traps are studied 
with a one dimensional single quantum well, which has the same effective mass as the surrounding semiconductor, while a MOS capacitor (MOS-CAP) system is used for analyzing oxide traps. The traps are assumed to be invariant planar structures in the other two dimensions.

The exact theory of the MTM method is detailed in Ref. 21. In this method, the 1D heterostructure is assumed to be composed of segments. Assuming for simplicity that the wavevector orthogonal component $\vec{k}_{\perp}=0$ in the extreme contact regions, the effective mass based 1D-heterostructure Schrödinger equation is solved for each segment, while taking into account the appropriate continuity conditions for each pair of subsequent segments. This set of equations is then transformed into self-contained segment matrices known as the transfer matrices. These matrices relate the wavefunction coefficients of subsequent segments. The asymptotic regions beyond the left-most and right-most segments are configured with a constant potential. Properly conditioning the behavior of the wavefunction in these asymptotic regions results in the so-called asymptotic Jost matrix. The determinant of the Jost matrix provides the resonant or quasi-bound states of the system. ${ }^{19}$

The flowchart in Fig. 1 represents the full numerical procedure. The energy range of interest, which spans over the electrostatic potential of a trap configuration, is an input to the solver. The characteristic roots of the asymptotic transfer matrix system can efficiently be determined from the iterative Newton-Raphson method and represent the trap energy level $E_{\mathrm{t}}$ (see Fig. 2). The correctness of the proposed numerical scheme is confirmed with the spectral values and the

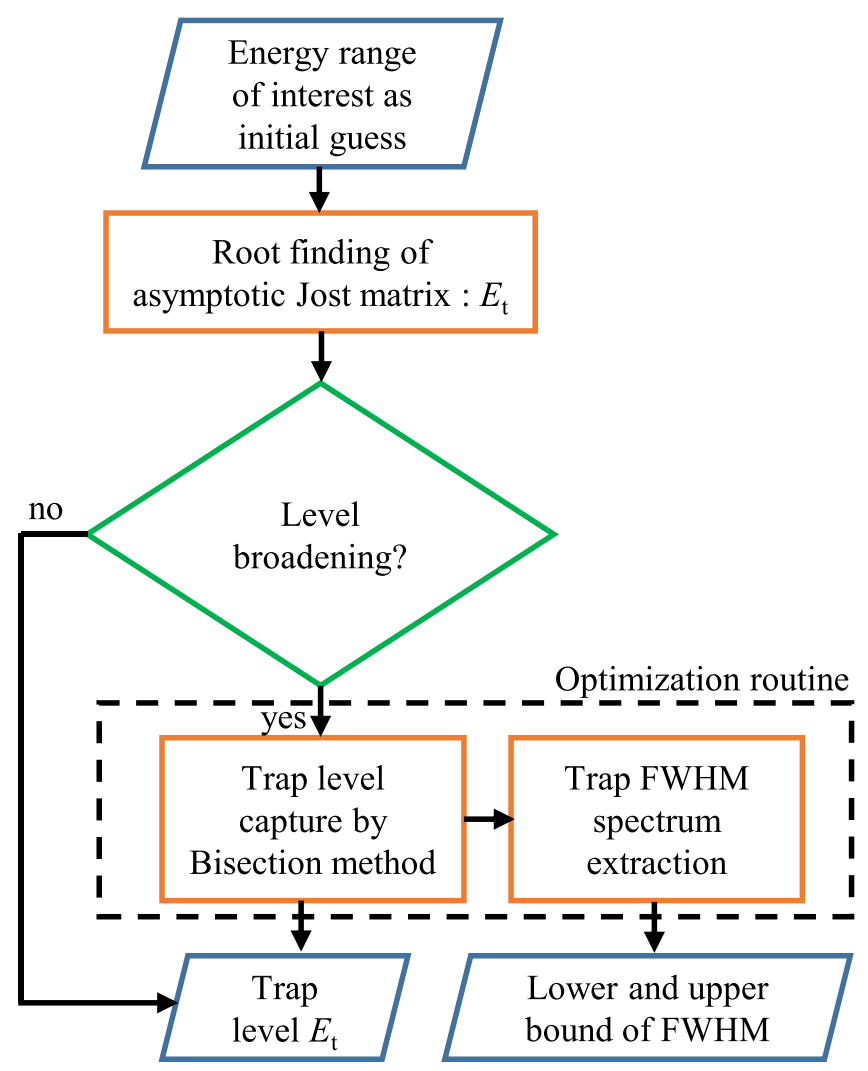

FIG. 1. Numerical scheme to find the trap level and FWHM spectral range.

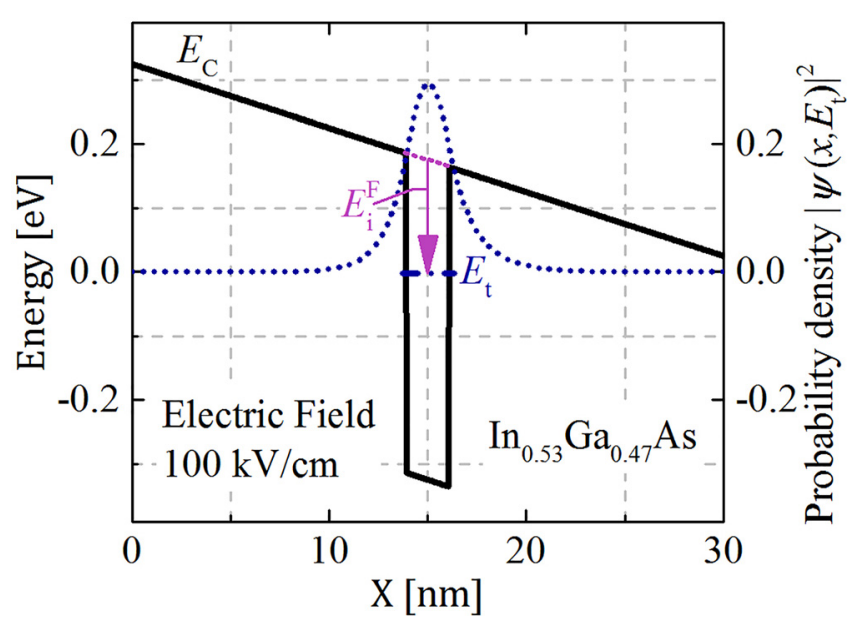

FIG. 2. The electrostatic potential (solid black), the characteristic level $\left(E_{\mathrm{t}} \approx-0.002 \mathrm{eV}\right)$, and the corresponding wavefunction of a $2 \mathrm{~nm}$-wide trap in a $30 \mathrm{~nm}$ long $\mathrm{In}_{0.53} \mathrm{Ga}_{0.47} \mathrm{As}$ region at $100 \mathrm{kV} / \mathrm{cm}$. $E_{\mathrm{i}}^{\mathrm{F}}$ is the energetic difference between $E_{\mathrm{C}}$ and $E_{\mathrm{t}}$, taken at the trap center.

corresponding wavefunction shapes of the resonant states of the heterostructures outlined in the literature. ${ }^{16,19}$

At high electric field, the broadening of a resonant state is expected. Therefore, the solver is extended with an optimization routine to determine the broadening of the trap energy at high fields. The solver calls for this routine only if the maximum probability density of the wavefunction inside the trap region has decreased with less than $50 \%$ at $1 \mu \mathrm{eV}$ from the extracted energy $E_{\mathrm{t}}$.

The optimization routine compares the wavefunction probability density peaks within the trap region and locates the energy interval corresponding to the full-width halfmaximum (FWHM). During this optimization routine, the imaginary component of the energy returned by the NewtonRaphson method is decreased to a negligible value in order to avoid an unphysical exponential increase of the planewave amplitude in the lower potential contact region (right contact in Fig. 2). To compare probability densities at different energies for quasi-bound systems, the assumption is made that the $E-k$ relation in the contact is linear in the range of trap levels of interest. Given the previous assumption that $\vec{k}_{\perp}=0$, and the typical proportionality $\left|E-E_{\mathrm{b}}\right|$ $\sim k^{2}$ (with $E_{\mathrm{b}}$ a band extremum), this assumption implies that the energy window of interest is far away from the material's band edge in the lower-potential contact region. Under these conditions, the state density at different energies is constant in the lower-potential contact region. The wavefunction densities are therefore normalized to this value. To compare probability densities in a bound system, deltanormalization is used.

\section{IMPACT OF ELECTRIC FIELD ON SEMICONDUCTOR TRAP LEVEL}

The impact of a TFET-like source-channel junction field on the trap is analyzed with different quantum well configurations in a uniform electric field. Fig. 2 illustrates the characteristic resonant state and the corresponding wavefunction of the square well (SW) trap structure at low 
uniform electric field $(F=100 \mathrm{kV} / \mathrm{cm})$. The system under consideration is a $30 \mathrm{~nm}$ long section of $\mathrm{In}_{0.53} \mathrm{Ga}_{0.47}$ As. At zero field, the arbitrarily chosen well depth and width are $0.5 \mathrm{eV}$ and $2 \mathrm{~nm}$, respectively. The first bound state of the system is at $169 \mathrm{meV}$ from the top of the conduction band $\left(E_{\mathrm{i}}=169 \mathrm{meV}\right)$ and could represent a shallow donor-like trap.

The sharp low-field energy level shown in Fig. 2 broadens into a spectral range $\left[E_{\mathrm{t}}^{\mathrm{L}}, E_{\mathrm{t}}^{\mathrm{U}}\right]$ at $750 \mathrm{kV} / \mathrm{cm}$ field strength as shown in Fig. 3(a). The densities in correspondence with the levels $E_{\mathrm{t}}^{\mathrm{Max}}, E_{\mathrm{t}}^{\mathrm{L}}$, and $E_{\mathrm{t}}^{\mathrm{U}}$ are illustrated in Fig. 3(b). It is evident from the comparison of Figs. 2 and 3 that this broadening signature can be attributed to the leakage of the trap wavefunction into the adjacent lower potential region (right side in Fig. 3).

For the SW trap configuration in Fig. 2, the electric field is varied from 0 to $3 \mathrm{MV} / \mathrm{cm}$. The resultant trap level shifts are plotted against the respective fields in Fig. 4. The level shift is calculated as the difference between the zero-field

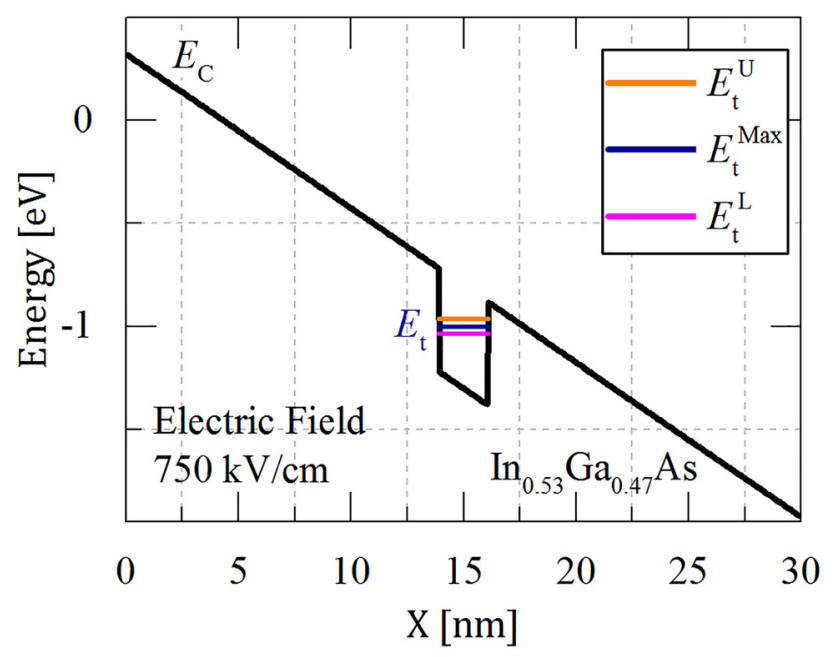

(a)

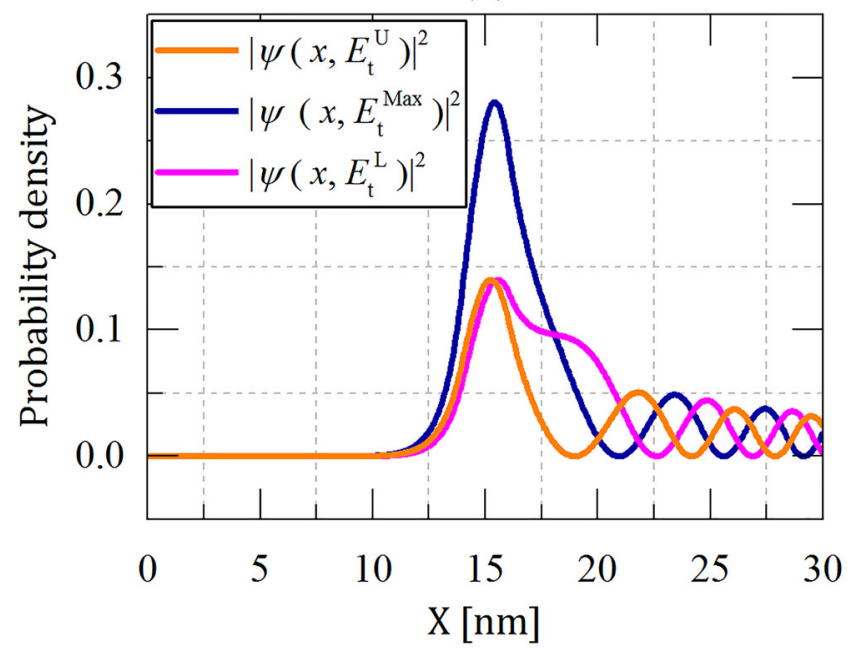

(b)

FIG. 3. (a) The electrostatic potential and the characteristic sub-levels $\left(E_{\mathrm{t}}^{\mathrm{Max}} \approx-1.003 \mathrm{eV}, E_{\mathrm{t}}^{\mathrm{L}} \approx-1.034 \mathrm{eV}\right.$ and $\left.E_{\mathrm{t}}^{\mathrm{U}} \approx-0.962 \mathrm{eV}\right)$, (b) The wavefunctions corresponding to the trap levels of (a), for a $2 \mathrm{~nm}$-wide trap in a $30 \mathrm{~nm}$ long $\mathrm{In}_{0.53} \mathrm{Ga}_{0.47} \mathrm{As}$ region.

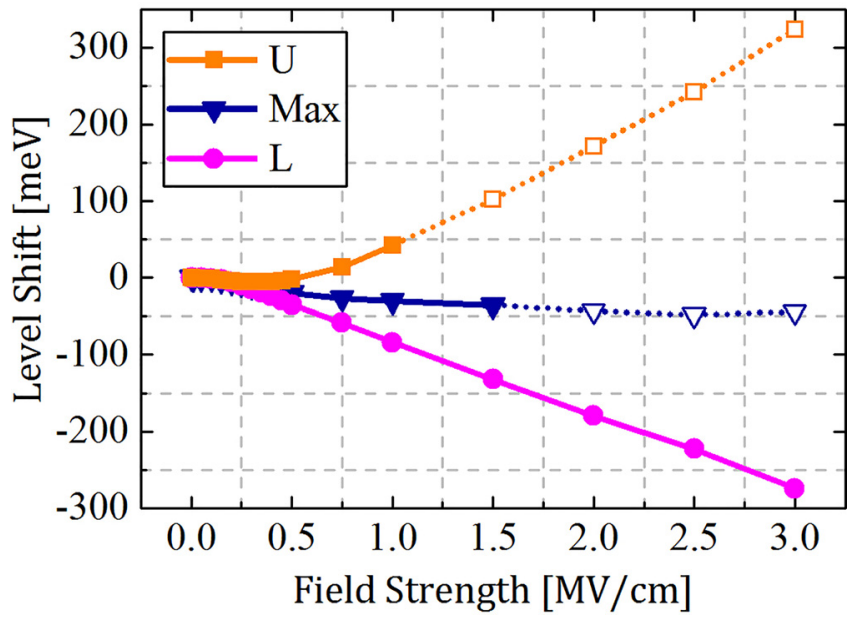

FIG. 4. Trap level shift and broadening as a function of electric field for the $2 \mathrm{~nm}$-wide SW of Fig. 2. The closed and open symbols represent the localization and non-localization (no energy barrier left at one side of the trap) of the trap-level $E_{\mathrm{t}}$ in the SW.

trap level $E_{\mathrm{i}}$ and the non-zero field trap level $E_{\mathrm{i}}^{\mathrm{F}}$, whereby the trap level $E_{\mathrm{i}}^{\mathrm{F}}$ is the energetic difference between $E_{\mathrm{C}}$ and $E_{\mathrm{t}}$, taken at the trap center (see Fig. 2). We show the shift of the most probable level (Max), the lower bound of the FWHM spectrum (L), and the upper bound of the FWHM spectrum (U).

In Fig. 4, the level broadening for the above trap configuration increases with electric field. Such signature is due to a reduction in potential barrier and hence an increase in leakage into the continuum states of the lower potential region. Additionally, the curve symbols change from closed to open as the probability density of the level becomes marginally peaked in the trap SW, this is, as the wavefunction becomes non-local. Note that the energy level corresponding to the maximum density need not be at the midpoint of its FWHM spectral range, as can be seen from Fig. 4.

Fig. 5 compares the level shift lines for a reduced width SW and a Coulomb well $(\mathrm{CW})$ with the previously defined $\mathrm{SW}$ (Fig. 2). Note that the energetic depth $E_{\mathrm{i}}^{\mathrm{F}}$ increases, as the depth as well as the width of the trap well increases. The energetic distance from the quantized energy level $E_{\mathrm{t}}$ to the bottom of the well increases with increasing trap depth and decreasing trap width. However, the different trap configurations are defined such that their zero-field trap energy levels $E_{\mathrm{i}}$ are identical (see inset of Fig. 5). It is apparent that the level shift and the level broadening depend on the barrier type. The $1 \mathrm{~nm}$ - wide trap encounters an increased effective barrier width and hence a smaller level shift than the $2 \mathrm{~nm}$ wide trap. The Coulomb trap experiences a hyperbolic barrier, which is smoother than the triangular barrier of SWs in an electric field. This results in a smaller effective barrier and hence a larger level broadening than that of a SW. From Fig. 5, the impact of well-known barrier lowering (PooleFrenkel effect) ${ }^{22}$ can also be seen as the early out-shift (open symbols) of the CW trap level compared to the SW level. Therefore, the particular choice of the trap potential ${ }^{23}$ in device electrostatics may have a quantitative impact on the predicted trap-based current. 


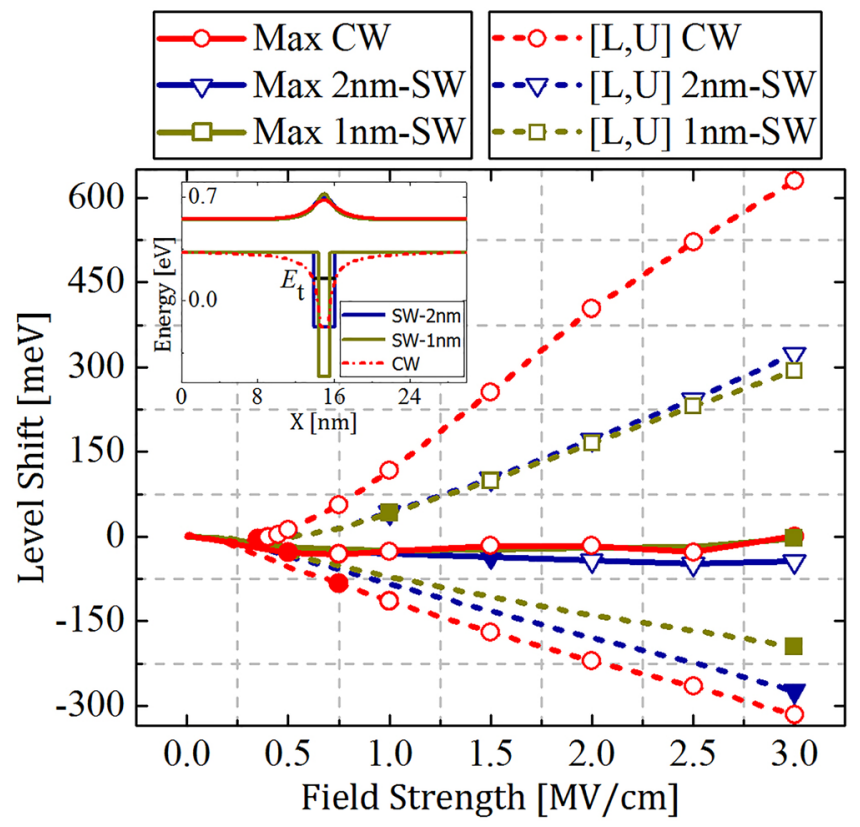

FIG. 5. Level shift and broadening as a function of electric field for the $2 \mathrm{~nm}$ wide SW, $1 \mathrm{~nm}$-wide SW, and the CW trap configurations. The open symbols represent the non-local trap states. The inset illustrates the described trap configurations at zero-field and the wavefunction probability densities for the indicated trap levels.

\section{ELECTRIC FIELD DEPENDENCE OF OXIDE TRAPS}

The formalism of the trap spectrum extraction (Fig. 1) is now used to determine the intrinsic characteristics of oxide traps in the presence of an electric field in a MOS-CAP system. In particular, we study the traps in $\mathrm{PolySi} / \mathrm{SiO}_{2} / \mathrm{p}^{-} \mathrm{Si}$ and $\mathrm{Al} /$ $\mathrm{HfO}_{2} / \mathrm{p}^{-} \mathrm{In}_{0.53} \mathrm{Ga}_{0.47} \mathrm{As}$ MOS heterostructures. In both of these structures, the substrate is doped with $10^{17} \mathrm{at} / \mathrm{cm}^{3}$ p-type concentration, while the oxide thickness and the substrate depth are $4 \mathrm{~nm}$ and $175 \mathrm{~nm}$, respectively (see Fig. 6). The trap in the oxide is configured with a constant $0.5 \mathrm{~nm}$ wide $\mathrm{SW}$. These MOS structures are biased from accumulation to inversion.

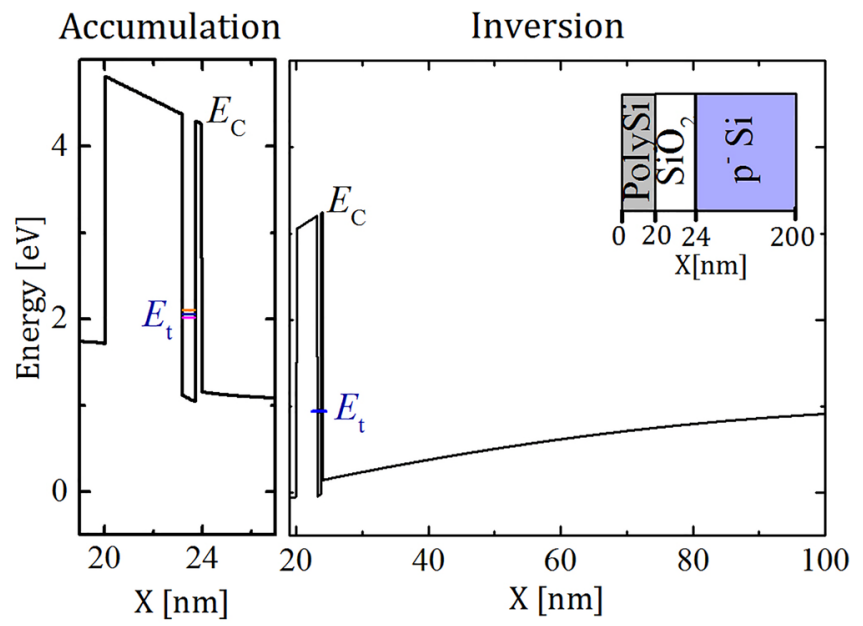

(a)

(b)

FIG. 6. A PolySi $/ \mathrm{SiO}_{2} / \mathrm{p}^{-} \mathrm{Si}$ MOSCAP with an oxide trap: (a) the conduction band energy in accumulation and (b) the conduction band energy in inversion. The oxide trap is configured as a $0.5 \mathrm{~nm}$ wide and $3.55 \mathrm{eV}$ deep SW and is located at $1 \mathrm{~nm}$ from the $\mathrm{SiO}_{2} / \mathrm{p}^{-} \mathrm{Si}$ interface.
For the PolySi $/ \mathrm{SiO}_{2} / \mathrm{p}^{-} \mathrm{Si}$ MOS system, the $0.5 \mathrm{~nm}$ wide $\mathrm{SW}$ type trap configuration is positioned at $1 \mathrm{~nm}$ from the $\mathrm{SiO}_{2} / \mathrm{p}^{-} \mathrm{Si}$ interface in the $\mathrm{SiO}_{2}$ (see Fig. 6). The depth of the well is varied from $2.05 \mathrm{eV}$ to $3.55 \mathrm{eV}$ with reference to the $\mathrm{SiO}_{2}$ conduction band edge. This combination of the MOS and the oxide trap structure is subjected to external applied biases. The resulting field induced trap level shifts and broadenings are plotted against the oxide field strength in Fig. 7. It is noticeable from this figure that the level shifts and level broadenings are quite low in comparison with the ones for the semiconductor traps of Fig. 5 due to the higher effective mass and barrier heights in the oxide $\left(E_{i}>2 \mathrm{eV}\right.$, $\left.m_{\mathrm{SiO}_{2}}^{*} \approx 0.5 m_{0}\right)^{24}$ compared to the semiconductor trap configurations $\left(E_{i}=0.17 \mathrm{eV}, m_{\mathrm{InGaAs}}^{*} \approx 0.043 m_{0}\right)$.

In accumulation, the levels shift in opposite direction to inversion. The negative level shift with increasing accumulation is due to the combined effects of the lowering of the substrate conduction band maximum (CBM) and lowering of the effective barrier height shown in Fig. 6(a). In contrast, the rise of the substrate $\mathrm{CBM}$ and the barrier height (see Fig. 6(b)) results in the positive level shift of the trap level with increasing depletion. The data in Fig. 7 also show that the level broadening is inversely related to the depth of the trap level, which is expected since deeper traps allow for less leakage.

The impact of the distance of the trap from the oxidesubstrate interface is illustrated in Fig. 8. The trap is configured with a constant $0.5 \mathrm{~nm}$ wide and $3.05 \mathrm{eV}$ deep $\mathrm{SW}$ inside $\mathrm{SiO}_{2}$. The position of the trap is varied in the range of $1.75 \mathrm{~nm}-0.25 \mathrm{~nm}$ from the $\mathrm{SiO}_{2} / \mathrm{p}^{-} \mathrm{Si}$ interface. It is evident from Fig. 8 that the level shifts and broadenings depend on the leakage of its wavefunction through the tunneling barrier: these field-induced quantum effects are more pronounced when the trap is positioned closer to the $\mathrm{SiO}_{2} / \mathrm{p}^{-} \mathrm{Si}$ interface.

Note that for these trap configurations, the trap level is found to be around $1 \mathrm{eV}$ above the substrate CBM at zerofield condition. As the bias brings the MOS capacitor more

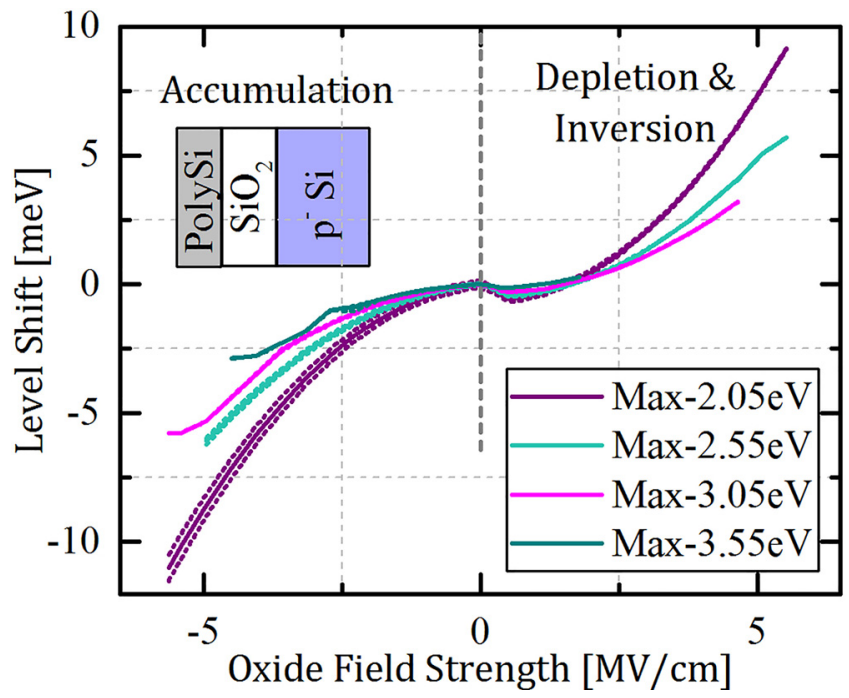

FIG. 7. Level shift (solid) and broadening (dashed) as a function of oxide electric field for the $0.5 \mathrm{~nm}$-wide $\mathrm{SW}$ oxide-trap configuration in the PolySi/ $\mathrm{SiO}_{2} / \mathrm{Si}$ MOS system. The traps are at $1 \mathrm{~nm}$ from the oxide-substrate interface and have varying well-depth configurations. 


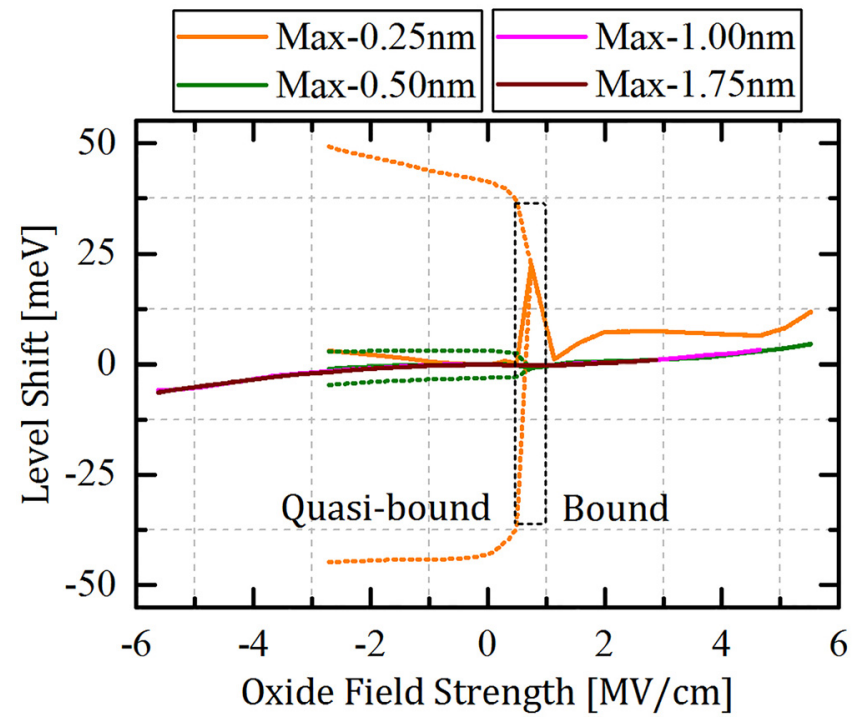

FIG. 8. Level shift (solid) and broadening (dashed) as a function of oxide electric field for the $0.5 \mathrm{~nm}$-wide $3.05 \mathrm{eV}$ deep $\mathrm{SW}$ oxide-trap configuration in the PolySi $/ \mathrm{SiO}_{2} / \mathrm{Si} \mathrm{MOS}$ system. The traps are at varying distance from the oxide-substrate interface. The trap-level is at around $1 \mathrm{eV}$ above the substrate conduction band edge at flat-band voltage condition. The black dashed box represents the transition from quasi-bound to bound systems.

in depletion, the trap level moves closer to and eventually below the substrate CBM. From the latter bias condition on, the trap level is fully localized (from a quasi-bound to a bound state as indicated in Fig. 8) and the level broadening disappears. The level values in the transition region indicated with a black dashed box are not reliable, as the approximation of fixed state density (in the lower potential contact region) no longer holds close to the conduction band edge. Hence, the comparison between the trap energy levels is no longer straightforward. The kinks observed in the depletion regime of the $0.25 \mathrm{~nm}$ spectral line are due to the coupling between the localized trap state and the quantized-inversion states in the substrate.

The oxide trap level characteristics also depend on the oxide material parameters such as the effective mass. An example is described in Fig. 9. With the $1 \mathrm{~nm}$ position away from the oxide-semiconductor interface of a $0.5 \mathrm{~nm}$ wide SW trap, the trap well-depths for both MOS systems in Fig. 9 are varied such that the energetic distance of the trap levels from the respective substrate conduction band edge is identical at zero-field condition. The substantial level shifts and broadenings of the $\mathrm{Al} / \mathrm{HfO}_{2} / \mathrm{In}_{0.53} \mathrm{Ga}_{0.47} \mathrm{As}$ MOS system can be ascribed to the lighter electron effective mass $\left(m_{\mathrm{HfO}_{2}}^{*}\right.$ $\left.\approx 0.11 m_{0}, m_{\mathrm{InGaAs}}^{*} \approx 0.043 m_{0}\right)$ than in $\mathrm{PolySi} / \mathrm{SiO}_{2} / \mathrm{p}^{-} \mathrm{Si}$ $\left(m_{\mathrm{SiO}_{2}}^{*} \approx 0.5 m_{0}, m_{\mathrm{Si}}^{*} \approx 0.09 m_{0}\right){ }^{24}$ The gradual disappearance of the level broadening by the depletion region and the corresponding inter-state coupling (between the bound trap and the bound substrate states) for the $\mathrm{Al} / \mathrm{HfO}_{2} / \mathrm{In}_{0.53} \mathrm{Ga}_{0.47} \mathrm{As}$ MOS system can also be noticed in Fig. 9.

\section{IMPACT OF LEVEL SHIFT ON THE EMISSION RATES OF TRAPS}

The field induced level shift and broadening will impact the prediction of trap related capture and emission rates in

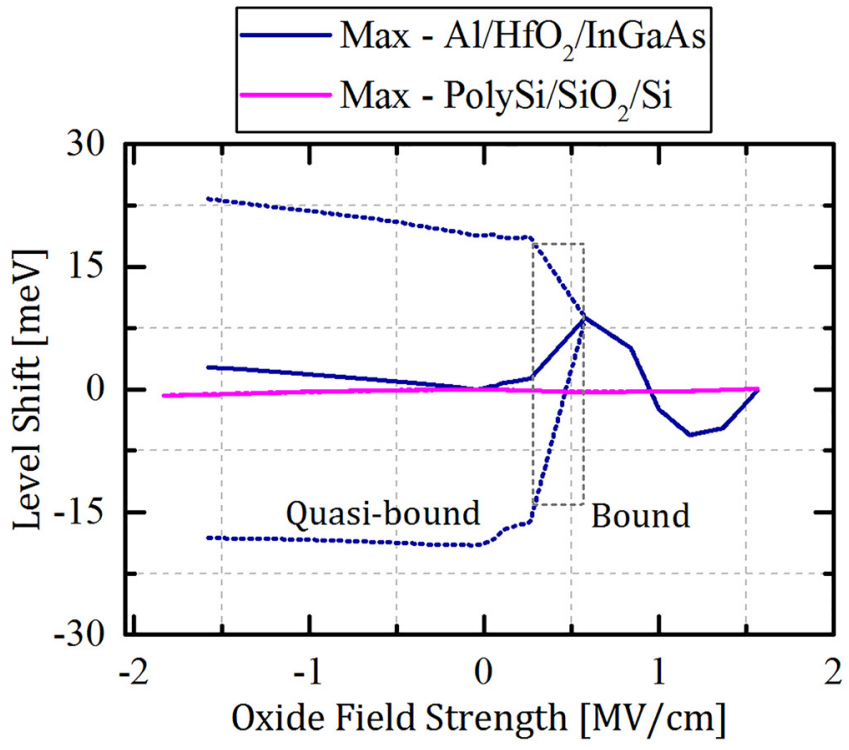

FIG. 9. Level shift (solid) and broadening (dashed) as a function of oxide electric field for the $0.5 \mathrm{~nm}$-wide SW oxide-trap configuration in PolySi/ $\mathrm{SiO}_{2} / \mathrm{Si}$ and $\mathrm{Al} / \mathrm{HfO}_{2} / \mathrm{InGaAs} \mathrm{MOS}$ systems. The traps are at fixed distance of $1 \mathrm{~nm}$ from the oxide-substrate interface and are with $3.05 \mathrm{eV}$ and $3.5 \mathrm{eV}$ well-depth configurations, respectively. The trap-levels in both MOS systems are at $1 \mathrm{eV}$ above the substrate conduction band edge in flat-band voltage condition. The quasi-bound to bound transition region is indicated with a black dashed box.

semiconductors and oxides as well as the calculation of TAT currents. One example is shown in Fig. 10, which graphically depicts the TFET electrostatics during the off-state. For the indicated mid-gap trap, the strong band bending owing to the high doping concentration in the TFET source can result in an apparent trap spectrum as opposed to the presumed sharp trap level in literature ${ }^{13,14}\left(E_{\mathrm{t}}\right)$. The lower bound of the spectrum can be responsible for larger TAT currents in TFETs, as the carriers can advance through this spectrum with higher probability since capture requires less thermal energy for a TAT event $(n<m)$, assuming that the tunneling process of the TAT event is very efficient.

In this article, we present first steps towards a TAT calculation by determining the semi-classical average emission rate from a trap in the presence of an electric field. In

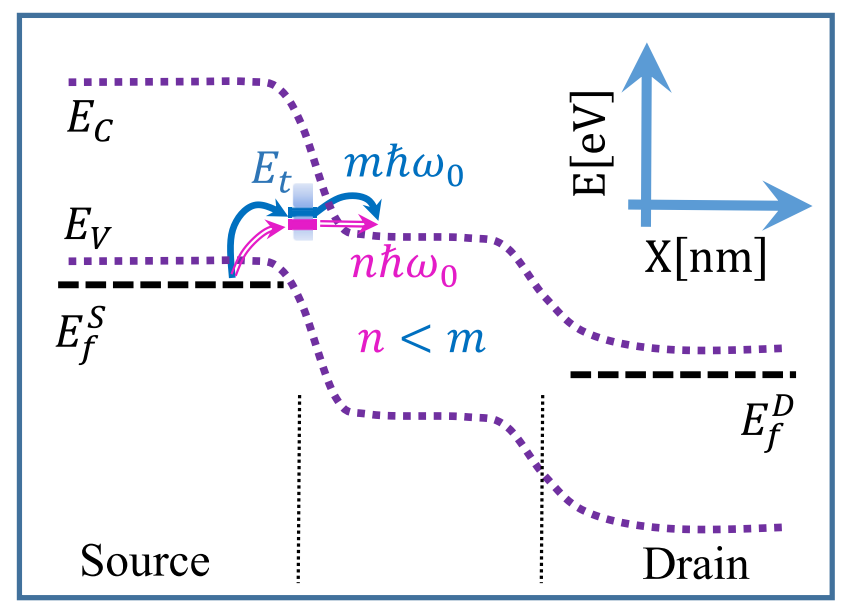

FIG. 10. Graphical illustration of the impact of electric field on the TAT in a simple p-i-n TFET in the off-state. 
TFETs, the TAT current associated with a specific trap state is proportional to its emission rate. We follow the work in the literature ${ }^{22,25}$ describing the field-dependent emission rates. The phonon-assisted emission rate formula for the SW and the $\mathrm{CW}$ is modified to account for the field-induced quantum effects on the trap level. The broadening of the trap level is implemented as a summation of emission rates weighted with probability density and normalized with the total sum of the probability densities of all sub-levels in the spectrum, whereby the spectrum is linearly discretized.

This results in the following average emission rate $\left\langle e_{n F}\right\rangle$ for the SW type semiconductor trap configuration with fieldinduced quantum effects (see also the inset to Fig. 11 for symbols' use)

$$
\frac{\left\langle e_{n F}\right\rangle}{e_{n 0}}=\frac{\sum_{\mu=\mathrm{L}}^{\mathrm{U}} \chi_{\mu} \exp \left\{\frac{\delta E_{\mu}}{k_{B} T}\right\}\left[1+\int_{0}^{E_{\mu} / k_{B} T} \mathrm{~d} z \exp \left\{z-z^{3 / 2}\left(\frac{4 \sqrt{2 m^{*}\left(k_{B} T\right)^{3}}}{3 q \hbar F}\right)\right\}\right]}{\sum_{\mu} \chi_{\mu}},
$$

where

$$
e_{n 0}=e_{n}^{\infty} \exp \left\{\frac{-E_{i}}{k_{B} T}\right\} ; \quad \chi_{\mu}=\left|\psi\left(x_{\mathrm{p}}, E_{\mu}\right)\right|^{2} ; \quad z=\frac{E_{\mu}-E_{t h}}{k_{B} T}
$$

and where $e_{n}^{\infty}\left(=\sigma_{n 0}\left\langle v_{t h}\right\rangle N_{D O S}^{C}\right)$ is the zero-field emission rate coefficient, $\sigma_{n 0}$ is the zero-field capture cross section, $\left\langle v_{t h}\right\rangle$ is the average thermal velocity of the carrier, $N_{D O S}^{C}$ is the effective density of states in the conduction band, $\chi_{\mu}$ is the probability density peak value for the trap sub-level $E_{\mu}$
( $x_{\mathrm{p}}$ being the position of the peak), $E_{\mu}$ is the trap sub-level at the trap center measured from $E_{C}, \delta E_{\mu}\left(=E_{i}-E_{\mu}\right)$ is the trap sub-level shift compared to the zero-field value, $E_{t h}$ is the net thermal energy which the carrier uses to emit from the trap, $k_{B}$ is the Boltzmann constant, and $T$ is the temperature. In Eq. (1), the exponential pre-factor $\left(\exp \left\{\delta E_{\mu}\right\}\right)$ reflects the dependence of the emission rates on the level shift and the summation over $\mu$ denotes the impact of broadening. Similarly, the average emission rate for the $\mathrm{CW}$ type trap can be extended as Eq. (2)

$$
\begin{aligned}
\frac{\left\langle e_{n F}\right\rangle}{e_{n 0}}= & \frac{\sum_{\mu=\mathrm{L}}^{\mathrm{U}} \chi_{\mu} \exp \left\{\frac{\delta E_{\mu}}{k_{B} T}\right\}\left[\exp \left\{\frac{\delta E_{i}^{P F}}{k_{B} T}\right\}\right]}{\sum_{\mu} \chi_{\mu}} \\
& +\frac{\sum_{\mu=\mathrm{L}}^{\mathrm{U}} \chi_{\mu} \exp \left\{\frac{\delta E_{\mu}}{k_{B} T}\right\}\left[\int_{\delta E_{i}^{P F} / k_{B} T}^{E_{\mu} / k_{B} T} \mathrm{~d} z \exp \left\{z-z^{3 / 2}\left(\frac{4 \sqrt{2 m^{*}\left(k_{B} T\right)^{3}}}{3 q \hbar F}\right)\left[1-\left(\frac{\delta E_{i}^{P F}}{z k_{B} T}\right)^{5 / 3}\right]\right\}\right]}{\sum_{\mu} \chi_{\mu}} .
\end{aligned}
$$

In Eq. (2), the barrier lowering $\left(\delta E_{i}^{P F}=q \sqrt{q F / \pi \varepsilon_{r} \varepsilon_{0}}\right)^{22}$ responsible for the Poole-Frenkel mechanism is unvaried with respect to the described effects. In the expression of $\delta E_{i}^{P F}, \varepsilon_{r}$ and $\varepsilon_{0}$ represent the relative permittivity of the host material at high frequency and the vacuum permittivity, respectively.

The emission rates of Eqs. (1) and (2) are applied to the $\mathrm{Cr}$ acceptor impurity ${ }^{22}$ with $\sigma_{n 0}=10^{-14} \mathrm{~cm}^{2}$ in $\mathrm{GaAs}$ (see inset of Fig. 11). This trap is configured as SW and $\mathrm{CW}\left(-Z q^{2} / 4 \pi \varepsilon_{r} \varepsilon_{0}|x|\right)$ with $Z=1, \quad \varepsilon_{r}^{\mathrm{GaAs}}=12.9 \varepsilon_{0}$. The ionization energy of this impurity in GaAs is $0.8 \mathrm{eV}$ and is considered to be the ground state of an isolated trap $\left(E_{\mathrm{i}}=0.8 \mathrm{eV}\right){ }^{22}$ Therefore, the $\mathrm{SW}$ is specified with an approximately one lattice constant $(0.6 \mathrm{~nm})$ wide and
$2.4 \mathrm{eV}$ deep specification, while the $\mathrm{CW}$ is configured as a $0.6 \mathrm{~nm}$ wide at the bottom and $2.8 \mathrm{eV}$ deep hydrogenic well. Fig. 11 replicates the results of phonon-assisted emission rates ${ }^{22}$ at room temperature $(300 \mathrm{~K})$, superimposed with the modified emission rates of Eqs. (1) and (2). Considering the impact of the level shift alone (replacing the summation of $\mu$ with Max in Eq. (1)), the emission rates are marginally impacted compared to the emission rates of the fixed $\mathrm{Cr}$ level for the SW (dashed blue and red emission lines in Fig. 11). The combined effect of level shift and spectral broadening can further enhance the emission rates for both trap configurations. This increase in emission rates is most pronounced in the high-field region and is negligible below $1 \mathrm{MV} / \mathrm{cm}$. 
The emission rate equations Eqs. (1) and (2) are limited to the triangular and hyperbolic barriers, respectively. In addition to this, the effective density of states into which the trap states can leak is assumed to be an abundant continuum of free states. However, the oxide-traps in a MOS capacitor system experience finite-width trapezoidal-like barrier structures. This example is illustrated in Fig. 12. For a SW-type oxide trap configuration of Fig. 12, the trapezoidal barrier can be treated as the combination of a triangular-type barrier and a fixed-width barrier. This results in the following emission rate expression for a fixed trap level: ${ }^{22}$

$$
\begin{aligned}
\frac{e_{n F}}{e_{n}^{\infty}}= & \exp \left\{\frac{-E_{i}}{k_{B} T}\right\}\left[1+\int_{0}^{E_{r} / k_{B} T} \mathrm{~d} z \exp \left\{z-z^{3 / 2}\left(\frac{4 \sqrt{2 m^{*}\left(k_{B} T\right)^{3}}}{3 q \hbar F}\right)\right\}\right] \\
& +\exp \left\{\frac{-E_{i}}{k_{B} T}\right\}\left[\int_{E_{r} / k_{B} T}^{E_{i} / k_{B} T} \mathrm{~d} z \exp \left\{z-\left(z+\frac{q F l_{t}}{2 k_{B} T}\right)^{1 / 2}\left(\frac{2 l_{t} \sqrt{2 m^{*} k_{B} T}}{\hbar}\right)\right\},\right.
\end{aligned}
$$

where $l_{t}$ is the tunneling length for a fixed-width barrier, $E_{r}$ is the energy at which the triangular barrier changes into a fixed-width barrier (see Fig. 12) measured from the oxide conduction band edge and at the center of the trap. The first integral of Eq. (3) corresponds to the emission through a triangular-type barrier. The emission through a fixed-width barrier is given by the second integral term of Eq. (3). This fixed-width barrier emission is based on the standard WKB-approximation for the tunneling probability through a finite barrier. In this integral term, the tunneling through a barrier is assumed to occur with an average imaginary wavevector ( $\mathrm{K}_{i}^{\text {av }}$ in Fig. 12) corresponding to the value of the imaginary wavevector at the center of the barrier. The field-induced level shift and the broadening is now added into Eq. (3) and results in the following average emission rate:

$$
\begin{aligned}
\frac{\left\langle e_{n F}\right\rangle}{e_{n}^{\infty}}= & \frac{\sum_{\mu=\mathrm{L}}^{\mathrm{U}} \chi_{\mu} \exp \left\{\frac{-E_{\mu}}{k_{B} T}\right\}\left[1+\int_{0}^{E_{r} / k_{B} T} \mathrm{~d} z \exp \left\{z-z^{3 / 2}\left(\frac{4 \sqrt{2 m^{*}\left(k_{B} T\right)^{3}}}{3 q \hbar F}\right)\right\}\right]}{\sum_{\mu} \chi_{\mu}} \\
& +\frac{\sum_{\mu=\mathrm{L}}^{\mathrm{U}} \chi_{\mu} \exp \left\{\frac{-E_{\mu}}{k_{B} T}\right\}\left[\int_{E_{r} / k_{B} T}^{E_{\mu} / k_{B} T} \mathrm{~d} z \exp \left\{z-\left(z+\frac{q F l_{t}}{2 k_{B} T}\right)^{1 / 2}\left(\frac{2 l_{t} \sqrt{2 m^{*} k_{B} T}}{\hbar}\right)\right\}\right]}{\sum_{\mu} \chi_{\mu}} .
\end{aligned}
$$

Note that Eq. (4) is used for finding the oxide trap emission rates which exhibits the level broadening at zero-field condition, whereas Eqs. (1) and (2) assume a sharp trap level for the semiconductor traps at zero-field condition.

The field dependent emission rates based on Eq. (4) for the oxide traps of PolySi $/ \mathrm{SiO}_{2} / \mathrm{p}^{-} \mathrm{Si}$ and $\mathrm{Al} / \mathrm{HfO}_{2} / \mathrm{In}_{0.53} \mathrm{Ga}_{0.47} \mathrm{As}$ MOS structures with $\sigma_{n 0}=10^{-15} \mathrm{~cm}^{2}$ at $300 \mathrm{~K}$ are shown in Fig. 13. The lower branch and the upper branch of each of the emission rate lines in Fig. 13 correspond to, respectively, the depletion and accumulation electrostatic domains of the considered MOS structures and reflect the impact of the effective barrier width towards the substrate. The different cases of the trap configuration are identical to the ones of Figs. 7, 8, and 9. Comparison with the fixed-trap level emission rate (determined with Eq. (3)) shows negligible difference, implying that the level shift and broadening have a negligible impact in the considered parameter space. From Fig. 13, the typical decrease of emission rates with the increase in trap energetic depth can be noticed. For the case of varying distance of the trap level from the $\mathrm{SiO}_{2} / \mathrm{p}^{-} \mathrm{Si}$ interface, the increase in emission rates with the decrease in barrier width is quite apparent in this figure. The effect of the decrease in tunneling length tends to decrease the split between the accumulation and depletion branches of emission lines. Additionally, Fig. 13 illustrates the improved emission rates for the $\mathrm{HfO}_{2}$ trap compared to that of $\mathrm{SiO}_{2}$, which can be related to the correspondingly lower effective mass in Eq. (4). Also for the $\mathrm{HfO}_{2} \mathrm{MOS}-\mathrm{CAP}$ configuration, the differences in the trap level shift and broadening (see Fig. 9) have a negligible impact on the emission rates.

Even though the emission rate model (Eqs. (3) and (4)) has the potential to include any trap-type configuration, it lacks the ability to account for the relaxation effects ${ }^{13,14,24}$ 


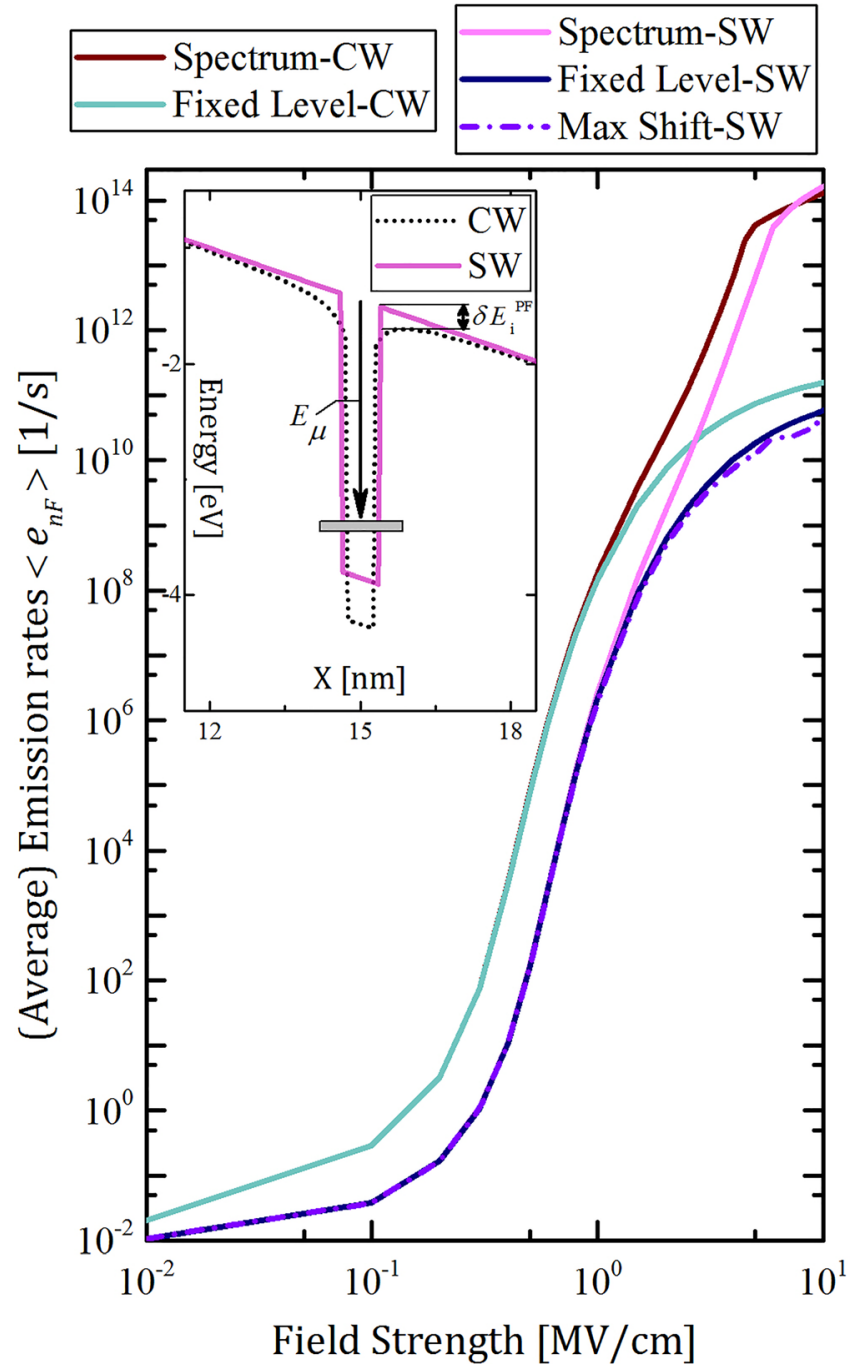

FIG. 11. Replication of the results outlined in Ref. 22 on a different scale, along with the derived intrinsic impact of the field on the emission rates. FL stands for fixed trap level, whereas the field effects are reflected in level shift-only (dash-dot) and the spectrum based emission lines.

associated with the trapping dynamics. However, this study provides a reference framework for the expected level shifts and broadenings, which could be incorporated into the effective capture and emission barrier heights in models including the relaxation effects. ${ }^{13,14,24}$

\section{CONCLUSIONS}

We explored the implications of high electric fields on the characteristic trap energy level. For the planar semiconductor and oxide traps in a one-dimensional structure, we outlined the numerical procedure to capture the field-induced level broadening, which is typically hundreds of $\mathrm{meV}$ for the former and tens of $\mathrm{meV}$ for the latter at electric fields of 2 $\mathrm{MV} / \mathrm{cm}$. The amount of broadening is sensitive to the chosen trap configuration and therefore future work should include realistic trap configurations. The field effects are implemented in the existing semi-classical emission rate formalism. We found that the field-induced quantum effects can increase the emission rates of a semiconductor trap level at high electric field, while the impact for the oxide traps is

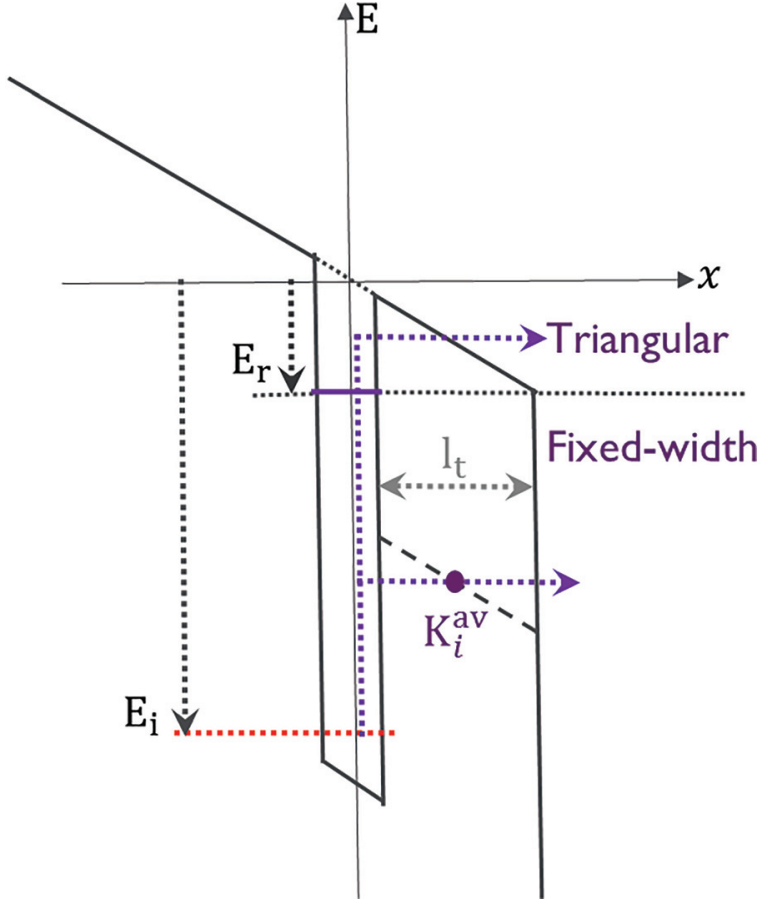

FIG. 12. Graphical illustration of the field enhanced emission mechanism in a finite-width barrier structure. The trapezoidal barrier is treated as a combination of a triangular-type and a fixed-width type barrier with a common ionization energy $\mathrm{E}_{r}$.

much smaller due to the higher effective mass and higher barrier heights than those in the former. In summary, the field-induced quantum effects for the trap level and the associated emission rates enhancement in a semiconductor

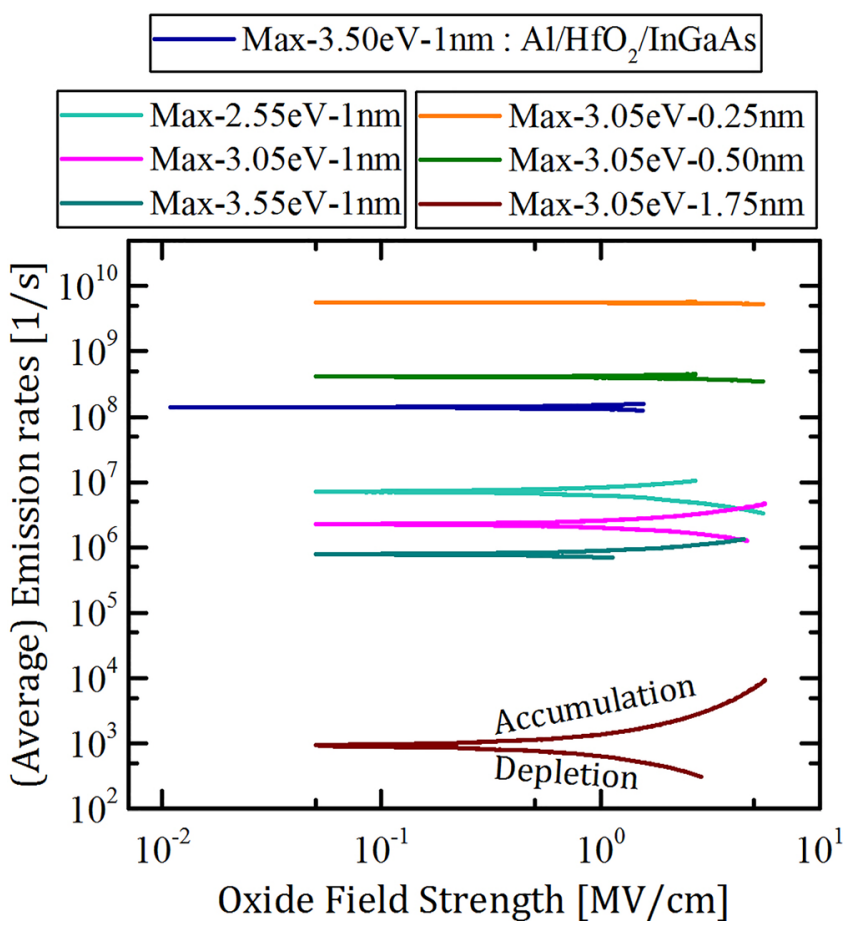

FIG. 13. Electric field enhanced average emission rates at $300 \mathrm{~K}$ as a function of oxide electric field for different instances of the $0.5 \mathrm{~nm}$-wide $\mathrm{SW}$ oxide-trap configuration in the $\mathrm{PolySi} / \mathrm{SiO}_{2} / \mathrm{Si}$ and the $\mathrm{Al} / \mathrm{HfO}_{2} /$ $\mathrm{In}_{0.53} \mathrm{Ga}_{0.47}$ As MOS systems. 
device depend on the combined influence of the field strength, trap charge, trap position, tunneling barrier type, and the host material parameters. It is expected that the broadening predicted in our article will impact the trapassisted tunneling currents but further study is needed to make quantitative statements.

\section{ACKNOWLEDGMENTS}

This work was supported by imec's Industrial Affiliation Program. D. Verreck acknowledges the support of a $\mathrm{PhD}$ stipend from IWT-Vlaanderen.

${ }^{1}$ H. Lu and A. Seabaugh, "Tunnel field-effect transistors: State-of-the-art," IEEE J. Electron Devices Soc. 2, 44-49 (2014).

${ }^{2}$ A. M. Ionescu and H. Riel, "Tunnel field-effect transistors as energyefficient electronic switches," Nature 479, 329-337 (2011).

${ }^{3}$ S. O. Koswatta, S. J. Koester, and W. Haensch, "On the possibility of obtaining MOSFET-like performance and sub-60-mV/dec swing in 1-D broken-gap tunnel transistors," IEEE Trans. Electron Devices 57, 3222-3230 (2010).

${ }^{4}$ D. Verreck, A. S. Verhulst, K. H. Kao, W. G. Vandenberghe, K. D. Meyer, and G. Groeseneken, "Quantum mechanical performance predictions of pn-i-n versus pocketed line tunnel field-effect transistors," IEEE Trans. Electron Devices 60, 2128-2134 (2013).

${ }^{5}$ D. Verreck, A. S. Verhulst, M. L. V. de Put, B. Sorée, N. Collaert, A. Mocuta, A. Thean, and G. Groeseneken, "Uniform strain in heterostructure tunnel field-effect transistors," IEEE Electron Device Lett. 37, 337-340 (2016).

${ }^{6} \mathrm{~K}$. Ganapathi and S. Salahuddin, "Heterojunction vertical band-to-band tunneling transistors for steep subthreshold swing and high on current," IEEE Electron Device Lett. 32, 689-691 (2011).

${ }^{7} \mathrm{M}$. Luisier and G. Klimeck, "Performance comparisons of tunneling fieldeffect transistors made of InSb, Carbon, and GaSb-InAs broken gap heterostructures," IEEE Int. Electron Devices Meet. (IEDM) 2009, 1-4.

${ }^{8}$ U. E. Avci, D. H. Morris, and I. A. Young, "Tunnel field-effect transistors: Prospects and challenges,” IEEE J. Electron Devices Soc. 3, 88-95 (2015). ${ }^{9}$ C. D. Bessire, M. T. Björk, H. Schmid, A. Schenk, K. B. Reuter, and H. Riel, "Trap-assisted tunneling in Si-InAs nanowire heterojunction tunnel diodes," Nano Lett. 11, 4195-4199 (2011).

${ }^{10}$ A. S. Verhulst, D. Verreck, Q. Smets, K. H. Kao, M. V. de Put, R. Rooyackers, B. Sorée, A. Vandooren, K. D. Meyer, G. Groeseneken, M. M. Heyns, A. Mocuta, N. Collaert, and A. V. Y. Thean, "Perspective of
tunnel-FET for future low-power technology nodes," IEEE Int. Electron Devices Meet. (IEDM) 2014, 30.2.1-30.2.4.

${ }^{11}$ J. Wu, L. F. Register, and E. Rosenbaum, "Trap-assisted tunneling current through ultra-thin oxide," in 37th Annual 1999 IEEE International Reliability Physics Symposium Proceedings (1999), pp. 389-395.

${ }^{12}$ Y. Mori, K. Takeda, and R.-i. Yamada, "Random telegraph noise of junction leakage current in submicron devices," J. Appl. Phys. 107, 014509 (2010).

${ }^{13} \mathrm{~A}$. Schenk, "A model for the field and temperature dependence of Shockley-Read-Hall lifetimes in silicon," Solid-State Electron. 35, 1585-1596 (1992)

${ }^{14}$ G. A. M. Hurkx, D. B. M. Klaassen, and M. P. G. Knuvers, "A new recombination model for device simulation including tunneling," IEEE Trans. Electron Devices 39, 331-338 (1992).

${ }^{15}$ V. L. Bonch-Bruevich and E. G. Landsberg, "Recombination mechanisms," Phys. Status Solidi B 29, 9-43 (1968).

${ }^{16}$ C. L. Fernando and W. R. Frensley, "An efficient method for the numerical evaluation of resonant states," J. Appl. Phys. 76, 2881-2886 (1994).

${ }^{17}$ W. R. Frensley, "Numerical evaluation of resonant states," Superlattices Microstruct. 11, 347-350 (1992).

${ }^{18}$ E. Anemogiannis, E. N. Glytsis, and T. K. Gaylord, "Quasi-bound states determination using a perturbed wavenumbers method in a large quantum box," IEEE J. Quantum Electron. 33, 742-752 (1997).

${ }^{19}$ S. A. Rakityansky, "Modified transfer matrix for nanostructures with arbitrary potential profile," Phys. Rev. B 70, 205323 (2004).

${ }^{20}$ L. L. Sánchez-Soto, J. J. Monzón, A. G. Barriuso, and J. F. Cariñena, "The transfer matrix: A geometrical perspective," Phys. Rep. 513, 191-227 (2012).

${ }^{21}$ S. A. Rakityansky, "Unified treatment of bound, scattering, and resonant states in one-dimensional semiconductor nanostructures," Phys. Rev. B 68, 195320 (2003).

${ }^{22} \mathrm{G}$. Vincent, A. Chantre, and D. Bois, "Electric field effect on the thermal emission of traps in semiconductor junctions," J. Appl. Phys. 50, 5484-5487 (1979).

${ }^{23}$ M. G. Pala, D. Esseni, and F. Conzatti, "Impact of interface traps on the IV curves of InAs tunnel-FETs and MOSFETs: A full quantum study," IEEE Int. Electron Devices Meet. (IEDM) 2012, 6.6.1-6.6.4.

${ }^{24} \mathrm{~T}$. Grasser, "Stochastic charge trapping in oxides: From random telegraph noise to bias temperature instabilities," in 2011 Reliability of Compound Semiconductors (ROCS) Workshop [Microelectron. Reliab. 52, 39-70 (2012)].

${ }^{25}$ P. A. Martin, B. G. Streetman, and K. Hess, "Electric field enhanced emission from noncoulombic traps in semiconductors," J. Appl. Phys. 52, 7409-7415 (1981). 